(31] C. L. Hakinson et L. S. Palmer. J. Dairy Sci., 1943, 26, 1043.

[32] H. Mattenheimer et Hs. Nitschmann. Helv. Chim. Acta, 1955, 38, 687.

[33] C. A. Zithle et J. Cerbulis. J. Dairy Sci, 1958, 41, 241.

[34] T. L. MoMemkin. In The Proteins, (Edit. E. Neurath et K. Bailey) New-York, 1954, vol. II, part. A.

[35] R. Aschaffenburg. J. Dairy Res., 1956, 23, 135.

[36] D. F. WaUgh et P. H, v. Hippel. J. Am. Chem. Soc., 1956, 78, 4576.

[37] T. L. MeMeenin, M. L. Groves et N. J. Hipp. 131 st. Meeting of the Am. Chem. Soc., 1957, 65 c.

[38] N. J. Hipp, M. L. Groves et T. L. McMenkin. 136st Meeting of the Am. Chem. Soc., 1959, 56.

[39] J. Long, Q. Winckel et I. A. Gould. J. Dairy Sci, 1958, 41, 317.

[40] D. F. WAUGH. Faraday Soc. Discussions, 1958, 25, 186.

[41] J. Garnier. Proc. Intern. Symposium Enzyme Chem., Tokyo, 1957, 525.

[42] R. G. WAKE. Austr. J. Science, 1957, 20, 167.

[43] T. Tsugo et K. Yamauchi. Bull. Agric. Chem. Soc. Japon, 1960, 24, 96.

[44] Hs. Nitschmann et R. Henzi. - Helv. Chim. Acta, 1959, 52, 1985.

[45] T. A. J. Payens. Biochim. Biophys. Acta, 1961, 46, 441.

\title{
CONTRIBUTION \\ A L'Étude de L'ACtivité Antibiotique DES SOUCHES DE CULTURE DU YOGHOURT ${ }^{(1)}$
}

\author{
par \\ J. RAŠIĆ et S. MITIĆ \\ Belgrade (Yougoslavie)
}

Le yoghourt et le lait caillé, en tant que produits, sont connus non seulement comme nutritifs et diététiques, mais aussi comme possédant des qualités de laits fermentés thérapeutiques. Déjà MeтchNikofF [1] avançait que l'effet inhibiteur du yoghourt sur certaines bactéries putrides et pathogènes, reposait non seulement sur l'acide lactique présent, mais aussi sur des matières spécifiques créées par $L b$. bulgaricus. C'est la raison pour laquelle ce chercheur recommandait que le yoghourt et le lait caillé soient utilisés dans l'alimentation en vue de la lutte contre les microorganismes putrides de l'intestin ou autres germes nuisibles.

(1) Ces recherches sont financées par les fonds pour les travaux scientifiques. 
Cela a incité les chercheurs ultérieurs à prêter plus d'attention à l'effet antagoniste des cultures de yoghourt. C'est ainsi que l'effet bactéricide et bactériostatique du yoghourt et du lait caillé, sur un nombre imposant de microorganismes pathogènes ou saprophytes, a été démontré par une série d'auteurs, confirmant de la sorte la présence de matières antibiotiques, d'un large éventail, dans ces laits fermentés [2], [3], [4], [5], [6], [7].

Emanuiloff et Natcheff [5], Fikov [8], Todorov [9], Schmidt et HANEMANN [10] nous renseignent également sur l'effet antagoniste des bactéries de yoghourt sur les germes pathogènes de l'intestin. L'effet bactéricide du yoghourt et du lait caillé sur les Mycobactéries a été remarqué et souligné par un certain nombre d'auteurs de divers pays [11], [12], [13]. Les expérimentations récentes, faites par TACQUET et coll. [14] montrent que les suspensions de Mycobactéries de type humain ou bovin, ainsi que du type BCG, mis dans le yoghourt, ont été anéanties aprés 18-24 heures, tandis que les Mycobactéries du type aviaire ou atypique sont restées en vie même après six jours de présence dans le yoghourt.

Nos propres recherches montrent une instabilité des matières antibiotiques du yoghourt pendant son exposition aux températures au-dessus de $0^{\circ} \mathrm{C}$, alors qu'aux températures très basses, telles que $-10^{\circ} \mathrm{C}$ et $-30^{\circ} \mathrm{C}$, l'activité antibiotique ne varie que très faiblement.

Le but de ces expérimentations a été de fixer les différences dans les activités antibiotiques des souches de diverses cultures de yoghourt (lactobacilles et streptocoques), recueillies dans les différentes régions de la Yougoslavie. Notre intention a été également de montrer quelles différences pouvaient exister dans l'activité antibiotique du yoghourt et celle des souches dont il est composé.

\section{EXPERIMENTATION}

Les cultures de yoghourt, objet de notre expérimentation, ont été choisies dans des échantillons de yoghourt et de lait caillé recueillis dans les différentes régions de la Yougoslavie. Lors de la composition et de l'assemblage des échantillons, nous avons tenu compte du fait qu'ils devaient présenter le yoghourt ou le lait caillé, produit de manière domestique avec les cultures autochtones de chaque région. Les échantillons ont été pris et placés dans des récipients stériles, aseptiques autant que possible, immédiatement réfrigérés et rapidement livrés au laboratoire.

Après leur isolement à partir des échantillons, les cultures ont été purifiées, identifiées et entretenues comme cultures pures dans 
du lait lavé stérile. Au total, il a été examiné et identifié 140 souches dont 100 appartenaient aux lactobacilles ( $L b$. bulgaricus et $L b$. jugurti), tandis que 40 souches appartenaient à l'espèce Str. thermophilus. Les résultats de ces recherches ont été publiés antérieurement [16].

En vue de l'examen de l'activité antibiotique, il a été utilisé 100 souches de lactobacilles et 19 souches de streptocoques. Les cultures testées ont été fournies par l'institut de l'usine de médicaments "Galenika » et sont d'origine américaine (collection de cultures pures). Ce sont les espèces suivantes : 1. Escherichia coli; 2. Staphylococcus aureus; 3. Candida albicans; 4. Staphylococcus oxford; 5. Bacillus subtilis.

Ces souches ont été entretenues sur gélose nutritive inclinée. Elles ont été régulièrement repiquées et, après incubation à $37^{\circ} \mathrm{C}$, conservées dans le réfrigérateur à la température de 4-6 ${ }^{\circ} \mathrm{C}$.

Pour la fixation du degré de l'activité antibiotique, on s'est servi de la méthode de dilution [5]. Lors de la préparation des échantillons, le chauffage a été fait dans l'autoclave suivant la méthode Tacquet et coll. [14]. L'ensemencement des éprouvettes d'expérimentation a été effectué par un inoculum contenant environ 1-1,5 million de cellules des souches choisies. Les cultures dont l'activité antibiotique s'était le mieux manifestée ont été l'objet d'examens continus par la méthode standard de diffusion sur gélose, à l'aide de cylindres en verre. Le diamètre de la zone d'inhibition a été mesuré au bout d'une période d'incubation de 16 à 18 heures et exprimé en millimètres. Toutes ces épreuves ont été l'objet de trois examens successifs.

Comme substrat pour les boîtes de Petri on a utilisé la gélose nutritive. Tous les éléments de ce substrat utilisé au cours des travaux d'expérimentation, avaient même origine. En outre il a été observé des conditions de travail, telles que la température et le temps d'incubation de la culture choisie, les cultures de yoghourt, les échantillons, la grandeur de l'inoculum etc, aussi semblables que possible.

\section{RÉSULTAT ET DISGUSSION}

\section{Activité antibiotique des souches de cultures de yoghourt}

Les résultats de l'examen de l'activité antibiotique des sou ches de lactobacilles et de streptocoques sont présentés dans le tableau I.

En étudiant ce tableau on peut constater que 30 p. 100 des 
TABLEAU I

AGTIVITÉ ANTIBIOTIQUE DES SOUGHES DE GULTURES DE YOGHOURT

\begin{tabular}{|c|c|c|c|c|c|c|c|c|c|c|c|c|c|c|c|c|c|c|c|c|}
\hline \multirow[t]{3}{*}{ Souches utilisées: } & \multicolumn{5}{|c|}{ Escherichia coli } & \multicolumn{5}{|c|}{ Staphylococcus aureus } & \multicolumn{5}{|c|}{ Candida albicans } & \multicolumn{5}{|c|}{ Bacillus subtilis } \\
\hline & \multicolumn{20}{|c|}{ Absence de croissance des filtrats des cultures de yoghourt à la dilution : } \\
\hline & 0 & $1: 2$ & $1: 4$ & $1: 8$ & $1: 16$ & 0 & $1: 2$ & $1: 4$ & $1: 8$ & $1: 16$ & 0 & $1: 2$ & $1: 4$ & $1: 18$ & $1: 16$ & 0 & $1: 2$ & $1: 4$ & $1: 8$ & $1: 16$ \\
\hline Lb. bulgaricus .... & 71 & 43 & 14 & 4 & 1 & 71 & 56 & 26 & 1 & 1 & 71 & 46 & 23 & 2 & - & 71 & 42 & 13 & 3 & 2 \\
\hline Lb. jugurti $\ldots \ldots$ & 29 & 28 & 18 & 3 & - & 29 & 28 & 24 & 2 & 1 & 29 & 29 & 21 & 2 & - & 29 & 28 & 16 & 4 & 1 \\
\hline Str. thermophilus . . & 19 & 1 & - & - & - & 19 & 5 & - & - & - & 19 & 14 & 7 & 5 & 2 & 19 & 15 & 7 & 3 & 2 \\
\hline
\end{tabular}


lactobacilles montrent un faible degré d'activité antibiotique, un moindre pourcentage $(3-7$ p. 100) dénote une activité très forte, tandis que le plus grand nombre des souches (environ 70 p. 100) se situe autour d'une activité moyenne.

Il est frappant de constater le nombre relativement faible de lactobacilles révélant une grande activité antibiotique. Cela nous montre toute la signification de la sélection des lactobacilles pour l'intensité de l'effet antibiotique sur les microorganismes pathogènes et saprophytes. Tant parmi les $L b$. bulgaricus que les $L b$. jugurti, il est des souches à forte et à faible activité antibiotique, fait qui nous amène à conclure que la sélection des cultures doit toujours comprendre les deux espèces de lactobacilles.

En 'comparant le degré d'acidité des souches respectivement avec l'activité antibiotique, il n'a pas été possible de remarquer une dépendance déterminée. Il est des souches avec un moindre degré d'acidité et avec une plus grande activité antibiotique et inversement. C'est ainsi que la souche $L b$. bulgaricus, marquée par $\mathrm{IIC}_{4}$, avait un moindre degré d'acidité - avec $84^{\circ}$ Thorner que la souche $\mathrm{Lb}$. bulgaricus $\mathrm{IIC}_{13^{-a v e c}} 100^{\circ}$ Thorner. Cependant la première montrait une activité antibiotique plus forte. Ou bien les souches $L b$, jugurti avec une acidité approximativement la même $\left(\mathrm{P}_{7}\right.$ et $\left.\mathrm{Z}_{6}\right)$ se différenciaient dans leur activité antibiotique de deux à quatre fois.

Les résultats de ces examens coïncident avec les constatations avancées par Dubinski [17], à savoir qu'il n'existe pas de dépendance déterminée entre l'acidité et la quantité de matières antibiotiques chez les différentes cultures de ferments lactiques.

Dans nos recherches antérieures [15], relatives à la réaction de l'activité antibiotique du yoghourt au cours de son exposition à des températures différentes, l'absence de dépendance déterminée entre le degré d'acidité et l'activité antibiotique du yoghourt a pu être également démontrée.

L'activité antibiotique des souches Str. thermophilus se différencie quelque peu des souches de lactobacilles (voir tableau I). C'est ainsi que l'on constate un degré plus faible d'activité antibiotique pour les cultures de Staphylococcus aureus et surtout pour les cultures Escherichia coli, par comparaison avec les lactobacilles. Les résultats de nos examens dans ce sens sont semblables à ceux de Todorov [9], qui a également montré l'effet inhibiteur moindre des streptocoques (Str. thermophilus) que des lactobacilles ( $L$ b. bulgaricus) sur les différents types de Salmonella lesquels sont, en tant que entero-bactéries, apparentées aux cultures d'Escherichia coli. 
Cependant, les souches de streptocoques ont montré approximativement la même ou un peu plus forte, activité antibiotique vis-à-vis des cultures utilisées telles que Bacillus subtilis et Candida albicans, que les lactobacilles, comme il est visible dans le tableau I.

Les différences constatées pour certaines souches de lactobacilles et de streptocoques, au cours de nos examens, dans l'activité antibiotique vis-à-vis des cultures, sont significatives du point de vue de la possibilité d'application de ces cultures à des fins thérapeutiques et technologiques.

\section{Activité antibiotique de divers yoghourts}

Pour obtenir une image aussi fidèle que possible de l'activité antibiotique de divers yoghourts, nous avons réuni les meilleures souches de lactobacilles et de streptocoques, obtenant ainsi onze sortes différentes de yoghourts. Ce faisant, nous avons tenu compte du fait que ces souches possédaient la même origine et présenté, dans chaque assemblage, le yoghourt (le lait caillé) d'une région. Pour pouvoir confirmer les résultats de l'examen de l'activité antibiotique des cultures de yoghourt à l'aide de la méthode de dilution, nous sommes allés plus loin en comparant nos résultats par l'application de la méthode de diffusion sur gélose. Les résultats de ces examens sont présentés par le tableau II.

TABLEAU II

AGTIVITÉ ANTIBIOTIQUE DE DIVERS YOGHOURTS

\begin{tabular}{|c|c|c|c|c|c|}
\hline \multirow[t]{2}{*}{$\begin{array}{c}\text { Désignation } \\
\text { des } \\
\text { Yoghourts }\end{array}$} & $\begin{array}{c}\text { Escherichia } \\
\text { coli }\end{array}$ & $\begin{array}{c}\text { Staphyl. } \\
\text { aureus }\end{array}$ & $\begin{array}{l}\text { Candida } \\
\text { albicans }\end{array}$ & $\begin{array}{c}\text { Bacillus } \\
\text { subtilis }\end{array}$ & $\begin{array}{l}\text { Acidité en } \\
\text { oThorner }\end{array}$ \\
\hline & \multicolumn{5}{|c|}{ Zone d'inhibition en $\mathrm{mm}$} \\
\hline Cet ...... & 0,5 & 0,5 & $0,5-1,0$ & $1,0-2,0$ & 76 \\
\hline Zet $\ldots \ldots$ & $2,0-3,0$ & $5,0-5,5$ & $3,0-4,0$ & $5,0-5,5$ & 93 \\
\hline $\mathrm{Zab} \ldots \ldots$ & 0,5 & $1,0-2,0$ & 1,0 & 1,0 & 84 \\
\hline G....... & $1,0-2,0$ & $3,0-4,0$ & $2,0-3,0$ & $2,0-3,0$ & 92 \\
\hline Vir. ..... & 0,5 & $0,5-1,0$ & 1,5 & 2,0 & 70 \\
\hline OH. ... & 2,0 & 3,0 & $1,0-2,0$ & $1,5-2,0$ & 95 \\
\hline $\mathrm{K} \ldots \ldots$ & $1,0-1,5$ & 2,0 & $1,0-1,5$ & $1,0-1,5$ & 93 \\
\hline $\mathrm{D}_{3} \ldots \ldots$ & $2,5-3,0$ & $4,0-5,0$ & $2,0-4,0$ & $4,0-5,0$ & 99 \\
\hline II $\mathrm{C} \ldots \ldots$ & $2,0-3,0$ & $4,0-5,0$ & $3,0-4,0$ & $5,0-6,0$ & 96 \\
\hline Str ..... & $1,0-2,0$ & $2,0-3,0$ & $3,0-4,0$ & $2,0-3,0$ & 90 \\
\hline $\mathrm{P} \ldots \ldots$ & $0,5-1,0$ & $1,0-2,0$ & $1,0-2,0$ & $1,0-1,5$ & 77 \\
\hline
\end{tabular}


Dans ce tableau, on peut voir clairement la différence qui existe entre les divers types de yoghourt du point de vue de l'effet antibiotique sur les cultures examinées. Si nous regardons la culture d'Escherichia coli, nous voyons que les yoghourts, marqués Zet, $\mathrm{D}_{3}$ et IIC, avec la zone d'inhibition de 2,0-3,0 $\mathrm{mm}$, ont montré une activité 4 à 6 fois plus grande que les yoghourts qui ont été les plus faibles à ce point de vue (Cet et Zab).

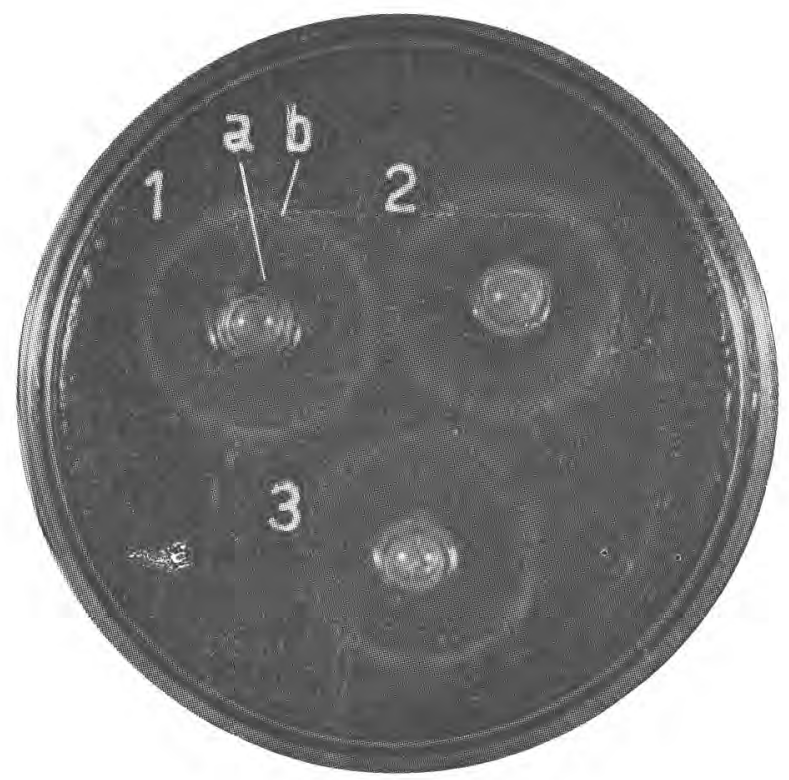

Fig. 1. - Activité antibiotique de divers yoghourts sur la culture Escherichia coli (Désignation des yoghourts : 1. - Zet.; 2. - II C. ; 3. - Vir.)

Ceci est également visible sur la figure 1. On y peut voir une chose intéressante. A côté de la zone d'inhibition — zone stérile, marquée par « $a$ » - on voit aussi une zone marquée par « b », où la croissance de la culture est bien plus fortement exprimée que sur les autres parties de la gélose nutritive dans la plaque de Petri. II semble que les matières antibiotiques du yoghourt diminuent en intensité, progressivement, lors de la diffusion dans la gélose, pour finir par se concentrer, sous une forme diluée, phénomène qui stimule la croissance des cellules d'Escherichia coli.

De même pour les autres cultures on remarque une inhibition différente de divers yoghourts. Ainsi la différence de l'activité 
antibiotique des meilleurs yoghourts (Zet, $D_{3}$ et IIC) chez la culture de Staphylococcus aureus a été évaluée à 8-10 fois par comparaison avec l'activité des échantillons de yoghourt les plus faibles (Cet. et Vir.). Ceci est également visible sur la figure 2.

Pour les cultures de Bacillus subtilis et de Candida albicans, on remarque la même différence entre les divers yoghourts du point de vue de l'effet antibiotique (fig. 3 et 4 ).

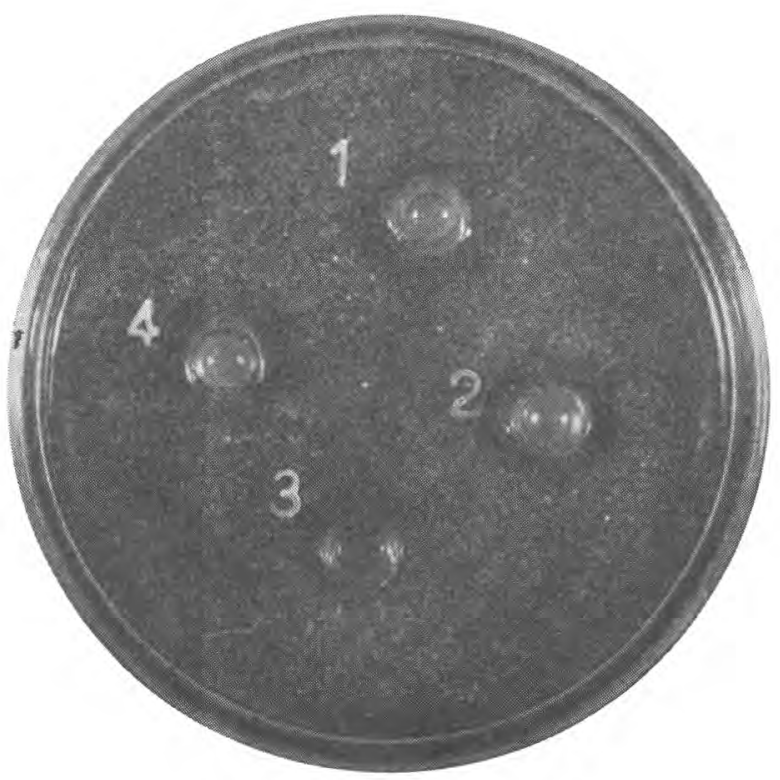

Fig. 2. - Activité antibiotique de divers yoghourts sur la culture de Staphylococcus aureus (Désignation des yoghourts : 1 . $-\mathrm{D}_{3} ; 2$. - Zet.; 3. - Str. ; 4. - P.)

Il va de soi que les différences entre les divers yoghourts seraient probablement plus fortement exprimées si l'on incluait dans l'examen de l'activité antibiotique d'autres cultures, lesquelles, ayant montré une faible activité, ont été éliminées de l'étude.

Par l'examen des différences de l'activité antibiotique des yoghourts et des souches diverses, il a été démontré que les yogourts manifestent une plus grande activité que n'importe quelle souche prise seule (Lb. bulgaricus ou Lb. jugurti et Str. thermophilus). L'effet antibiotique exprimé par les souches de lactobacilles pour les cultures d'Escherichia coli, de Staphylococcus aureus et par les 


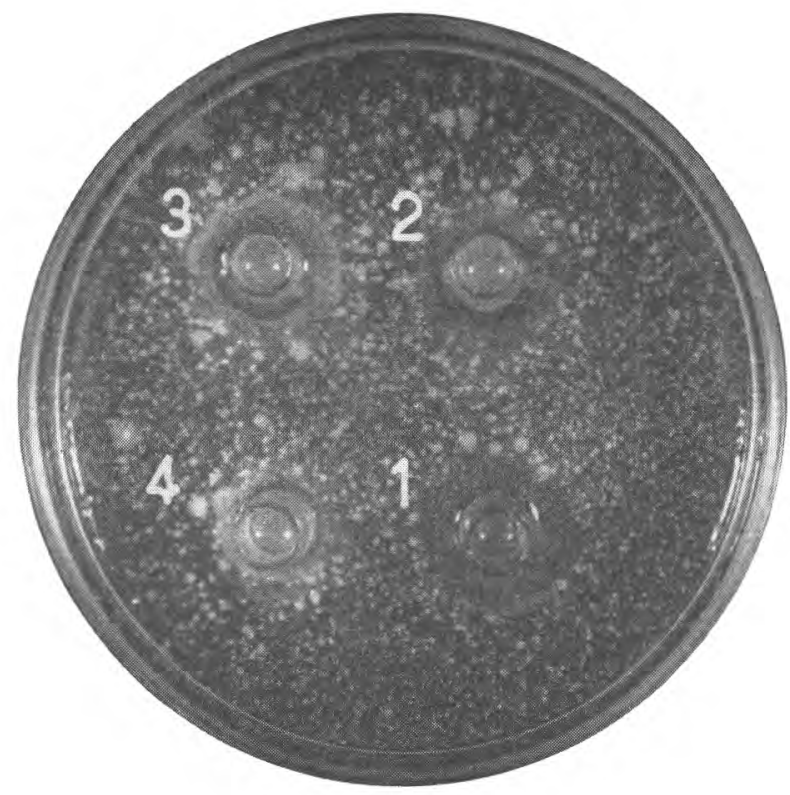

Fig. 3. - Activité antibiotique de divers yoghourts sur la culture de Bacillus subtilis (Désignation des yoghourts : 1. $-\mathrm{D}_{3}$.; 2. - Str. ; 3. - P.; 4. - Vir.)

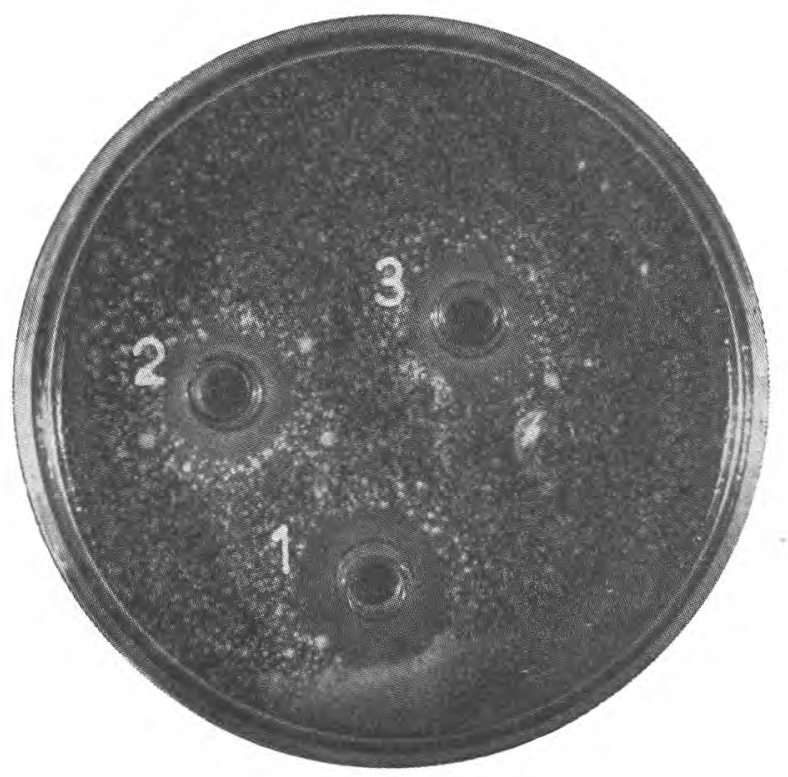

Fig. 4. - Activité antibiotique de divers yoghourts sur la culture de Bacillus subtilis. (Désignation des yoghourts : 1. — Zet.; 2. - G. ; 3. - Str.) 
streptocoques vis-à-vis de Candida albicans et de Bacillus subtilis, a pu utilement se combiner et trouver son expression dans le yoghourt (le lait caillé). Si l'on prend en considération la symbiose remarquée de lactobacilles et de streptocoques dans le yoghourt [18], il est alors facile de comprendre l'effet antibiotique accru du yoghourt par comparaison avec les souches isolées dont il est issu.

Les résultats de nos examens montrent clairement l'importance de la sélection des souches pour yoghourts d'après leur activité antibiotique, étant donné l'usage toujours plus grand du yoghourt et du lait caillé pour l'alimentation des hommes, ainsi que pour des fins diététiques et thérapeutiques.

\section{RÉSUMÉ}

Les cultures utilisées pour la fabrication du yoghourt se différencient considérablement entre elles du point de vue de l'activité antibiotique. Les souches de lactobacilles ( $L b$. bulgaricus et $L b$. jugurti) ont montré un plus grand degré d'activité antibiotique vis-à-vis des cultures d'Escherichia coli et de Staphylococcus aureus que les souches de streptocoques (Str. thermophilus), tandis que pour les cultures de Bacillus subtilis et de Candida albicans, les lactobacilles ont montré la même, ou un peu plus faible, activité par comparaison avec les souches de streptocoques.

On a remarqué chez les lactobacilles, tant parmi les souches de $L b$. bulgaricus que parmi les souches de $L b$. jugurti que l'on rencontre des cultures de différentes activités, ce qui nécessite une sélection attentive suivant leurs propriétés antibiotiques.

On na pas remarqué de relation précise entre le degré d'acidité et l'activité antibiotique des diverses souches.

Les yoghourts ont montré une activité antibiotique plus grande vis-à-vis des cultures éprouvées que chacune des souches dont le yoghourt est issu.

\section{SUMMARY}

Investigations were made on the antibiotic activity of yoghurt cultures collected from different parts of the country. It has been investigated a total of 119 strains. The results of investigations may be summarised as follows:

There are considerable differences among the yoghurt cultures in their antibiotic activities.

The strains of $L b$. bulgaricus and $L b$. jugurti show a greater degree of antibiotic activity to the test cultures of Escherichia-coli and Staphylococcus aureus, than the Str. thermophilus strains. Contrary to this, lactobacilli showed the same or weaker antibiotic 
activity to the test cultures of Bacillus subtilis and Candida albicans than the Str. thermophilus strains.

There is no definite relationship between the acid production and antibiotic activity with various cultures.

Yoghurt showed a greater antibiotic activity to the investigated test cultures than each strain (Str. themophilus and Lb. bulgaricus or $L b$. jugurti) which composed the yoghurt as a finished product.

The significance of demonstrated differences among the strains of yoghurt bacteria has been briefly discussed.

\section{BIBLIOGRAPHIE}

[1] Metschnikoff, I. Ann. de l'Inst. Pasteur, 1908, 12, 929.

[2] Miň́ev, P. Vet. sbirka, 1930, 5, 6.

[3] Seneca, H., Henderson, E. and Collins, A. Amer. Pract. and Dig. Treatment, 1950, 1, 1252.

[4] Bogdanov, I. Naucni trud. ISUL, 1952, 1, Nr. 2.

[5] Emanuiloff, I. und. Natscheff. L. Int. Milchvv. kongr., 1956, 1, 548.

[6] Brochu E., Riel R. et Vezina C., Les Bactéries lactiques et les laits fermentés. Le Conseil des Recherches Agricoles, Ministère de l'Agriculture de la Province de Québec. Nr. 3, 1959.

[7] Nikolov, N. M. Blgarsko kiselo mljako i drugi mlecnokiseli produkti. Zemizdat. Sofija, 1962.

[8] Fikov A. Kiselo mljako i izpolzuvaneto mu pri dietetikata i lekuvaneto na krmačetata. Lekkoopizdat. Sofija, 1945.

[9] Todonov, D. Proc. Int. Assoc. Vet. Fd. Hyg., 1960, 366. Dairy Sci. Abs., 24, 1716.

[10] Sсhмidt B., und Hannemann G., Z. Hyg. Infrekt. Kranh, 1959, 145, 499.

[11] Katrandziev, K. Izv. Mikr. Inst. BAN, 1951, Nr. 2.

[12] Gursel, A. et Nursret, F. Turk ijiyen Tecrubi Biol. Dergisi, 1953, 12, 79.

[13] Gursel, A. Turk ijiyen Tecrubi Biol. Dergisi, 1957, 17, 40.

[14] Tacquet A., Tison F. et Devulder B., Ann. de l'Inst. Pasteur, 1961, $100,581$.

[15] RAŝsic J. (sous presse).

[16] RAŝIC J. i. Mitic, S. Prehranb. ind., 1962, 12, 2374.

[17] Dubinski, R. Mol. Prom., 1957, 3, 39.

[18] Petre J. W. and Lolkema H., Neth Milk et Dairy J., 1950, 4, 197. 